\title{
Análise qualitativa do efeito da terapia por contensão induzida em crianças com paralisia cerebral
}

\section{Qualitative analysis of the effect of constraint induced movement therapy in children with cerebral palsy}

\author{
Liz Araújo Rohr ${ }^{1}$, Camila Araújo Santos Santana ${ }^{1}$, Erika Shirley Moreira da Silva ${ }^{1}$ \\ Carolina Daniel de Lima-Alvarez ${ }^{2}$, Gabriela Lopes dos Santos-Maia ${ }^{1}$, Eloisa Tudella ${ }^{1}$
}

http://dx.doi.org/10.11606/issn.2238-6149.v30i2p132-139

Rohr LA, Santos CAS, Silva ESM, Lima-Alvarez CD, Santos-Maira GL, Tudella E. Análise qualitativa do efeito da terapia por contensão induzida em crianças com paralisia cerebral. Rev Ter Ocup Univ São Paulo. 2020 maio-ago.;30(2):132-39.

RESUMO: Este estudo descreve quatro casos de pacientes com paralisia cerebral hemiparética, com idade entre quatro e onze anos $(6,50 \pm 3,32)$, que realizaram um protocolo de Terapia por Contensão Induzida (TCI). O objetivo foi analisar qualitativamente o efeito da TCI no uso do membro superior acometido. O protocolo foi realizado durante três semanas, onde o membro não acometido foi contido por gesso sintético e foram realizadas atividades funcionais com a transferência dos ganhos ocorridos durante o tratamento para o ambiente real. A análise qualitativa foi realizada por meio dos ajustes distais do membro acometido e por meio da escala Quality of Upper Skills Test (QUEST). Os quatro participantes obtiveram melhora nos ajustes distais e na pontuação da escala aplicada. A terapia por contensão induzida pode ser uma estratégia de intervenção eficaz para crianças com paralisia cerebral hemiparética.

DESCRITORES: Modalidades de fisioterapia; Paralisia cerebral; Criança; Pré-escolar.
Rohr LA, Santos CAS, Silva ESM, Lima-Alvarez CD, Santos-Maira GL, Tudella E. Qualitative analysis of the effect of constraint induced movement therapy in children with cerebral palsy. Rev Ter Ocup Univ São Paulo. 2020 May-Aug.;30(2):132-39.

ABSTRACT: This study describes the results of four children with hemiparetic cerebral palsy, age between four and eleven years (6.50土 3.32) who had performed a Constraint-induced Movement Therapy (CIMT) protocol. The purpose was to analyze qualitatively the effects of the CIMT protocol on the use of the affected upper limb. The protocol was performed for three weeks, with restriction of the nonaffected upper limb and functional activities were performed with the transfer of gains during treatment to the real environment. A qualitative analysis of the upper limb movement was performed through the Quality of Upper Skills Test (QUEST) scale and the assessment of distal adjustments. Improvements on the QUEST scale score and on the distal adjustments were observed in all four participants after the training protocol. The constraint-induced movement therapy may be an effective intervention aiming to improve the quality of the upper limb movement in children with hemiparetic cerebral palsy.

KEYWORDS: Physical therapy modalities; Cerebral palsy; Child; Child preschool

1. Departmento de Fisioterapia, Universidade Federal de São Carlos. São Carlos, SP, Brasil. ORCID: Rohr LA: https:/orcid.org/ 0000-0002-9566-1228; Santana CAS - https://orcid.org/0000-0003-1383-8366; Silva ESM- erikashirley.fisioped@gmail.com; Santos-Maia GL - https://orcid.org/0000-0002-6230-999X; Tudella E - https://orcid.org/0000-0003-0824-7350. Email: lizrohr.nenem@ gmail.com; camilaaraujo.s@hotmail.com; erikashirley.fisioped@gmail.com; gabrielalsantos@hotmail.com; tudella@terra.com.br.

2. Universidade Federal do Rio Grande do Norte - Natal/RN- Brasil. ORCID: https://orcid.org/0000-0002-2126-2937. Email: caroldaniellima@gmail.com

Endereço para correspondência: Liz A. Rohr. Universidade Federal de São Carlos: Rodovia Washington Luís, km 235. São Carlos, SP. CEP: 13565-905. E-mail: lizrohr.nenem@gmail.com (Liz Araújo Rohr). 


\section{INTRODUÇÃO}

$\mathrm{O}$ comprometimento motor é uma das principais características das crianças com Paralisia Cerebral (PC), o qual influencia o desempenho funcional dessas crianças ${ }^{1}$. Uma das complicações mais frequentes, ocorrendo em cerca de $29 \%$ dos casos, é a hemiparesia espástica ${ }^{2}$, que compromete um hemicorpo, com prejuízo mais acentuado no membro superior ${ }^{3}$.

Crianças com PC hemiparética frequentemente apresentam limitações na capacidade de alcançar, segurar e manipular objetos ${ }^{4}$. Déficits sensoriais, espasticidade e a fraqueza muscular do membro superior mais afetado limitam a exploração, o autocuidado e execução das atividades cotidianas com este membro ${ }^{5}$, de forma que a criança realiza suas tarefas motoras com o membro não afetado, o que caracteriza o fenômeno descrito como desuso aprendido ${ }^{6}$. $\mathrm{O}$ desuso aprendido consiste na redução do uso do membro superior acometido nas atividades cotidianas e é considerado uma alteração comportamental devido à dificuldade de executar movimentos com o braço, o que compromete a qualidade e a frequência dos movimentos da extremidade contralateral à lesão ${ }^{7}$.

A fisioterapia tem papel fundamental a fim de minimizar o impacto dessas alterações nos pacientes com PC. Nesse sentido, dentre as modalidades de intervenção disponíveis, a terapia de contenção induzida (TCI) tem demonstrado bons resultados. Essa técnica foi desenvolvida em 1980 por Edward Taub, inicialmente para pacientes com alterações motoras do membro superior decorrentes de acidente vascular encefálico ${ }^{8}$.

Estudos que aplicaram protocolos de TCI têm evidenciado resultados positivos em relação à funcionalidade da mão e do membro superior mais acometido em crianças com PC P $^{4,910,11}$. Porém, ainda faz-se necessário estudos que explorem os efeitos da TCI na qualidade do movimento em diferentes idades e seu efeito a longo prazo.

Com o intuito de agregar conhecimento no que diz respeito às estratégias de intervenção na área da reabilitação neuroinfantil, e colaborar com evidências científicas sobre intervenções que possam melhorar a funcionalidade, participação social e qualidade de vida de crianças com paralisia cerebral, o presente estudo teve como objetivo verificar qualitativamente o efeito de um protocolo de TCI no desempenho funcional e nos ajustes distais do membro superior comprometido em crianças com paralisia cerebral hemiparética.

A hipótese da pesquisa é que após o protocolo da TCI ocorreriam melhoras na qualidade do movimento e funcionalidade do membro superior hemiparético, e que estes efeitos seriam mantidos ao logo do tempo após terapia.

\section{PROCEDIMENTOS METODOLÓGICOS}

O presente estudo foi aprovado pelo Comitê de Ética e Pesquisa em Seres Humanos da Universidade Federal de São Carlos (1.171.889), onde as avaliações e tratamento foram realizadas no ano de 2017.

Participaram deste estudo 4 crianças com idade entre quatro e onze anos $(6,50 \pm 3,32)$, sendo três do sexo masculino e uma do sexo feminino, diagnosticadas com paralisia cerebral hemiparética espástica que eram atendidas no ambulatório de neuropediatria da Universidade Federal de São Carlos (UFSCar), na Unidade de Saúde Escola (USE) da UFSCar-campus São Carlos.

Os pais ou responsáveis foram contatados por telefone, informados sobre os objetivos e os procedimentos do estudo. Os pais ou responsáveis que aceitaram participar do presente estudo assinaram o Termo de Consentimento Livre e Esclarecido o qual está de acordo com a Declaração de Helsinki e com a resolução 466/2012 do Conselho Nacional de Saúde.

Os participantes foram classificados de acordo com o Sistema de Classificação da Função Motora Grossa (GMFCS) e pelo Sistema de Classificação da Habilidade Manual (MACS) (Tabela 1). Todos os participantes deste estudo atenderam os critérios para a realização da TCI conforme estabelecido por Winstein e colaboradores ${ }^{12}$.

Tabela 1 - Caracterização dos sujeitos de acordo com idade, peso, altura, GMFCS e MACS dos participantes do estudo

\begin{tabular}{ccccccc}
\hline Participante & Sexo & Idade (anos) & Peso (kg) & Altura (m) & GMFCS & MACS \\
\hline 1 & M & 4 & 21.00 & 1.06 & I & III \\
2 & M & 7 & 23.20 & 1.16 & II & III \\
3 & F & 4 & 13.30 & 1.00 & I & III \\
4 & M & 11 & 40.8 & 1.42 & II & III \\
& Média & $\mathbf{6 , 5 0}$ & $\mathbf{2 4 , 5 7}$ & $\mathbf{1 , 1 6}$ & & - \\
& DP & $\mathbf{3 , 3 2}$ & $\mathbf{1 1 , 6 2}$ & $\mathbf{0 , 1 8}$ & & \\
\hline
\end{tabular}

$\mathrm{DP}=$ desvio padrão 


\section{Procedimentos de avaliação}

As avaliações, do pré e pós tratamento ocorreram no Laboratório de Pesquisas e Análise do Movimento (LaPAM), integrado ao Núcleo de Estudos em Neuropediatria e Motricidade (NENEM), do departamento de Fisioterapia da Universidade Federal de São Carlos (UFSCar) campus São Carlos-SP.
Em no máximo sete dias após a pré-avaliação foi dado início ao protocolo de TCI na casa do participante. Posteriormente ao tratamento, os participantes foram submetidos as reavaliações que aconteceram nos seguintes momentos: 24 horas, 10 dias e 2 meses após o término do tratamento.

As avaliações e o protocolo de TCI foram realizadas por fisioterapeutas com experiência na área da reabilitação pediátrica.

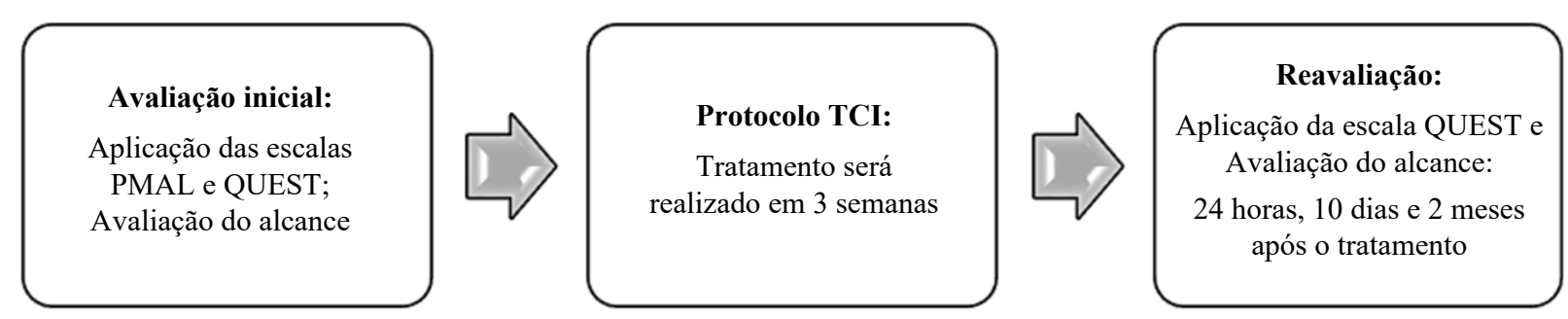

Figura 1 - Protocolo experimental

\section{Quality of Upper Extremit Skills Test (QUEST):}

A escala Quality of Upper Extremit Skills Test (QUEST), foi aplicada em todas as avaliações, e tem por objetivo de avaliar a qualidade do movimento dos membros superiores em crianças com PC de quatro domínios: dissociação de movimentos (1), preensões (2), extensão protetiva (3) e transferência de peso (4). A qualidade é definida com base na pontuação obtida em cada domínio, e a pontuação total é o resultado da soma de todas as pontuações de domínio divididas pelo número de domínios testados realizados, sendo que as pontuações mais altas indicam melhor qualidade do uso do membro superior ${ }^{13}$. No presente estudo avaliou-se os domínios 1, 2 e 3 .

\section{Análise qualitativa do alcance}

Para avaliação da tarefa de alcance, o participante foi posicionado sentado em uma cadeira de frente para uma mesa na qual suas mãos estavam apoiadas e um copo posicionado ao centro. Foi instruído aos participantes que realizassem a tarefa de alcançar e apreender o copo, leválo até a boca e devolvê-lo na mesa. Foram posicionadas três câmeras para filmar o movimento, sendo uma câmera anterior e duas laterais à mesa de teste. Cada participante realizou uma tentativa de treino e em seguida três tentativas de teste com o membro mais acometido.

O desempenho durante o movimento de alcance foi utilizado para avaliar os ajustes distais, que envolvem os ajustes feitos pelas mãos e dedos durante a trajetória do movimento de alcance. No presente estudo foram analisados os seguintes ajustes distais: orientação da palma da mão, que corresponde a posição da mão no momento em que toca o objeto e pode ser classificada em: mão horizontal, oblíqua ou vertical ${ }^{14}$; superfície de contatos da mão e dedos, que avalia a superfície da mão e dedos que tocam o objeto no momento do alcance, a qual pode ser classificada em ventral ou dorsa ${ }^{15}$; abertura da mão no momento do toque, a qual avalia a posição da mão no momento do alcance e pode ser classificada em: mão aberta, semiaberta ou fechada ${ }^{16}$, e preensão, onde será considerado alcance com preensão quando a criança apreender o objeto ou parte sem deixá-lo cair imediatamente ${ }^{16}$.Com relação a variável preensão, os autores classificaram os alcances em preensão com ajuda do membro não acometido (MNA) e sem ajuda.

\section{Protocolo de TCI}

Após a primeira avaliação foram feitas as medidas para confecção do gesso de contensão para cada paciente (do braço mais funcional). Em no máximo sete dias após a pré-avaliação foi dado início ao protocolo de TCI na casa do participante, com duração de três semanas, com treino intensivo durante cinco dias consecutivos por três horas por dia.

A escala Pediatric Upper Extremity Scale Motor Activity $\log$ (PMAL) foi aplicada na primeira avaliação para verificar as atividades mais comprometidas e determinar a partir destas as que seriam realizadas diariamente durante $o$ protocolo. Assim, as atividades realizadas durante o protocolo foram elaboradas individualmente para cada criança e 
tinham como objetivo aumentar o uso do membro mais comprometido em tarefas funcionais, como por exemplo, a alimentação e vestimenta. As atividades realizadas seguiram os três princípios da TCI, o primeiro corresponde ao treino orientado a tarefa com progressão da dificuldade desta tarefa (task pratice e shaping), o segundo refere-se a contensão do membro superior menos acometido e o terceiro corresponde a transferência dos ganhos ocorridos durante o tratamento para o ambiente real ${ }^{17}$.

Além disso, os pais ou responsáveis foram orientados em relação a um diário de tarefas. As tarefas diárias foram determinadas de acordo com o interesse e a necessidade de cada criança a partir dos resultados da PMAL e foi orientado para os pais para aplicarem essas atividades no dia a dia da criança, onde estes anotavam quais atividades a criança conseguiu realizar.

\section{RESULTADOS}

As variáveis deste estudo foram avaliadas através de uma análise descritiva dos resultados obtidos, em porcentagem. $\mathrm{O}$ índice de concordância inter-observador foi de $83,33 \%$ o qual foi realizado através do seguinte cálculo:

$$
\begin{aligned}
& \frac{\mathrm{N}^{\mathrm{o}} \text { concordância }}{\mathrm{N}^{\mathrm{o}} \text { concordância }}+\underset{+}{+} \\
& \mathrm{N}^{\mathrm{o}} \text { discondância }
\end{aligned}
$$

\section{Orientação da palma da mão}

Ao analisarmos a variável orientação da palma da mão, observamos que na avaliação inicial o participante 1 realizou 50\% dos alcances com a mão na posição horizontal e $50 \%$ com a mão oblíqua. No pós imediato realizou $60 \%$ do alcances com a mão oblíqua e no pós 10 dias $100 \%$ dos alcances foram realizados com a mão oblíqua. No que se refere a avaliação após 2 meses o participante realizou todos os alcances com a mão na horizontal (Tabela 2).

O participante 2 na avaliação inicial realizou $66,66 \%$ dos alcances com a mão na horizontal e no pós imediato realizou 50\% dos alcances com a mão oblíqua e 50\% com a mão na vertical. No pós 10 dias 66,66\% dos alcances foram realizados com a mão oblíqua e $33,34 \%$ com a mão na horizontal. Na última avaliação, o participante teve resultados similares a avaliação pós 10 dias e realizou $50 \%$ dos alcances com a mão oblíqua e 50\% com a mão na horizontal (Tabela 2).

$\mathrm{Na}$ avaliação inicial, o participante 3 realizou todos os alcances com a mão na posição vertical. No pós-imediato, pós 10 dias e pós 2 meses, todos os alcances foram realizados com a mão oblíqua (Tabela 2 ).

O participante 4 , na avaliação inicial realizou $50 \%$ dos alcances com a mão horizontal e 50\% com a mão oblíqua. No pós-imediato e pós 10 dias foi observado que o participante realizou $66 \%$ dos alcances com a mão oblíqua e na última avaliação os alcances foram realizados com a mão na posição oblíqua $(66,66 \%)$ e vertical $(33,34 \%)$.

\begin{tabular}{|c|c|c|c|c|c|c|c|c|c|c|c|c|}
\hline & \multicolumn{12}{|c|}{ Orientação da palma da mão, no membro acometido (\%) * } \\
\hline & \multicolumn{3}{|c|}{ Pré avaliação } & \multicolumn{3}{|c|}{ Pós imediato } & \multicolumn{3}{|c|}{ Pós 10 dias } & \multicolumn{3}{|c|}{ Pós 2 meses } \\
\hline & $\mathbf{H}$ & $\mathbf{O}$ & $\mathbf{V}$ & $\mathbf{H}$ & $\mathbf{O}$ & $\mathbf{V}$ & $\mathbf{H}$ & $\mathbf{O}$ & $\mathbf{V}$ & $\mathbf{H}$ & $\mathbf{O}$ & $\mathbf{V}$ \\
\hline $\mathbf{P 1}$ & 50 & 50 & 0 & 40 & 60 & 0 & 0 & 100 & 0 & 100 & 0 & 0 \\
\hline $\mathbf{P 2}$ & 66,66 & 33,34 & 0 & 0 & 50 & 50 & 33,34 & 66,66 & 0 & 50 & 50 & 0 \\
\hline P3 & 0 & 0 & 100 & 0 & 100 & 0 & 0 & 100 & 0 & 0 & 100 & 0 \\
\hline P4 & 50 & 50 & 0 & 33,34 & 66,66 & 0 & 33,34 & 66,66 & 0 & 0 & 66,66 & 33,34 \\
\hline
\end{tabular}

Tabela 2 - Orientação da palma da mão durante a realização dos alcances no membro acometido

$\mathrm{P} 1=$ participante $1 ; \mathrm{P} 2=$ participante $2 ; \mathrm{P} 3=$ participante $3 ; \mathrm{P} 4=$ participante $4 ; \mathrm{H}=$ mão horizontal; $\mathrm{O}=$ mão oblíqua; $\mathrm{V}=$ mão Vertical. *Todos os dados estão descritos em \%.

\section{Superfície de contado da mão e dedos}

Não foram observadas diferenças entre as avaliações, uma vez que todos os participantes tocaram o copo com a superfície ventral da mão e dedos em todas as avaliações.

\begin{abstract}
Abertura da mão
Ao observarmos a abertura da mão no momento do toque (Tabela 3), nota-se que na avaliação inicial todos os participantes realizaram os alcances com a
\end{abstract}


mão e dedos abertos. Já na avaliação do pós imediato o participante 4 realizou $33,3 \%$ dos alcances com a mão e dedos semiabertos e $66,6 \%$ com a mão e dedos abertos. Nas avaliações pós 10 dias e pós 2 meses os participantes 2, 3 e 4 realizaram o alcance com a mão e dedos abertos, e o participante 1 que na avaliação após 2 meses, realizou $67 \%$ dos alcances com a mão e dedos semiabertos e $33 \%$ com a mão e dedos abertos.

Tabela 3 - Abertura da mão no momento do toque membro acometido.

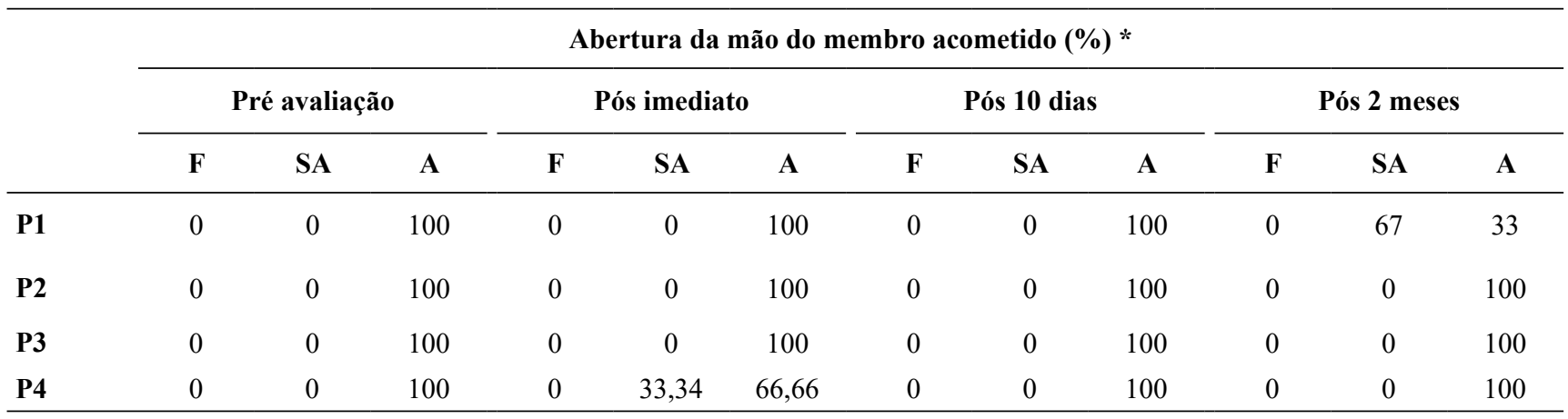

$\mathrm{P} 1=$ participante $1 ; \mathrm{P} 2=$ participante $2 ; \mathrm{P} 3=$ participante $3 ; \mathrm{P} 4=$ participante $\mathrm{F}=$ mão fechada; $\mathrm{SA}=$ mão semiaberta; $\mathrm{A}=$ mão aberta. *Todos os dados estão descritos em \%.

\section{Preensão}

Em relação a variável preensão, os alcances foram classificados em preensão com ajuda do membro não acometido (MNA) e sem ajuda. Pode-se observar que a quantidade de alcances realizados com a ajuda do MNA foi superior aos alcances realizados sem ajuda. Os participantes 2 e 3 obtiveram uma melhora nesta variável, sendo que o participante 2 realizou $50 \%$ dos alcances sem ajuda no pós imediato e no pós 2 meses. Já o participante 3 obteve uma melhora na avaliação do pós 10 dias, onde realizou $50 \%$ dos alcances foram realizados sem ajuda, porém este resultado não se manteve na avaliação após 2 meses realizando $100 \%$ dos alcances com ajuda do MNA.

\section{Quality of Upper Extremity Skill Test (QUEST)}

Os resultados obtidos através da escala Quest mostram que todas as crianças melhoraram sua pontuação nos domínios avaliados após o término da terapia. Dentre estes, pode-se observar que o domínio de preensão foi o que teve maior acréscimo na pontuação, exceto para o participante 4. Observa-se também que para o domínio dissociação de movimentos o participante 3 não manteve os ganhos obtidos nas duas últimas avaliações (após 10 dias e após 2 meses do término da terapia) e o participante 1 não manteve os ganhos obtidos no domínio extensão protetiva, pontuando o mesmo escore na pré-avaliação e na avaliação após 2 meses do término da terapia (Tabela 4).

Tabela 4 - Resultados escala Quest

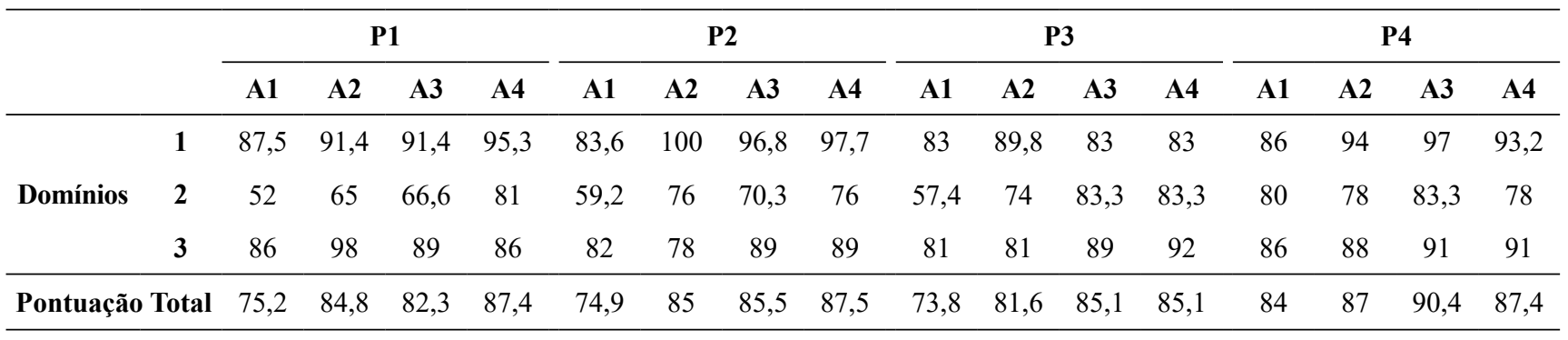

$\mathrm{A} 1=$ pré avaliação; $\mathrm{A} 2=$ pós imediato; $\mathrm{A} 3$ = pós 10 dias; $\mathrm{A} 4=$ pós 2 meses. $\mathrm{P} 1=$ participante $1 ; \mathrm{P} 2=$ participante $2 ; \mathrm{P} 3=$ participante $3 ; \mathrm{P} 4=$ participante 4 . Domínio 1= dissociação de movimento; Domínio 2= preensões; Domíno 3= extensão protetiva. 


\section{DISCUSSÃO}

O presente estudo teve como objetivo, descrever qualitativamente o efeito de um protocolo de TCI realizado com quatro crianças com paralisia cerebral hemiparética e com idade entre quatro e onze anos.

Os resultados deste estudo demostram que o protocolo de TCI promoveu melhora da qualidade do movimento da mão do membro hemiparético, a qual foi observada por meio das mudanças nos ajustes distais de orientação da palma da mão. As crianças realizaram mais alcances com a mão na posição oblíqua ou na vertical após o protocolo de treino, as quais são consideradas mais funcionais para a atividade que foi avaliada. Estes resultados podem ser explicados pelas características do protocolo, como a especificidade da tarefa, intensidade, e frequência do tratamento. Além disso, as atividades realizadas durante o tratamento foram direcionadas para a necessidade de cada criança e eram tarefas funcionais, possibilitando a transferência para o ambiente real da criança, como por exemplo atividades que simulavam a alimentação.

Nota-se que as crianças mais novas não realizaram nenhum alcance com a mão na posição vertical nas avaliações pós-tratamento. No presente estudo, as crianças mais novas foram mais resistentes ao tratamento, o que pode ter prejudicado a participação durante a terapia. Estes resultados contrapõe os achados de outros autores ${ }^{18}$, que por meio de uma revisão sistemática concluíram que a TCI traz benefícios em relação a atividade e participação das crianças com PC e que a idade não influencia no efeito da terapia.

Observamos também melhora na frequência dos alcances sem ajuda do membro mais funcional nos participantes 2 e 3 , o que demonstra a contribuição do protocolo de TCI para o uso funcional do membro superior mais acometido nesses pacientes. Porém, de modo geral entre os participantes, a quantidade de alcances realizados com ajuda do membro não acometido ainda continuou superior aos alcances realizados sem ajuda. A atividade de apreender um copo e levá-lo a boca pode ser complexa de ser realizada com o membro acometido para crianças com PC. Para apreender o copo a criança precisa realizar componentes de movimentos complexos e que não envolve somente movimentos das articulações do punho e dedos, mas também componentes de movimento das articulações de cotovelo e ombro. Estudos demonstram que crianças com PC utilizam movimentos compensatórios de tronco ${ }^{19}$ e apresentam limitação nos movimentos de extensão de cotovelo e supinação durante uma tarefa de alcançar ${ }^{19,20}$. Ademais, crianças com PC apresentam pobre coordenação motora, apresentando menor velocidade média, pico de velocidade e índice de retidão, mais unidades de movimentos e maior duração de movimento, durante uma tarefa de beber suco de um copo ${ }^{21}$.

Desta forma, concluímos que a TCI parece ser uma modalidade de terapia que pode melhorar qualidade do alcance das crianças com PC hemiparética e para que estes benefícios sejam potencializados sugerimos que durante a realização deste protocolo sejam realizadas atividades que trabalhem os componentes de movimentos citados acima.

Ademais, a melhora da função do alcance pode ter um impacto positivo na participação das crianças com PC. Pashmdarfard e Shervin ${ }^{22}$ demostram em seu estudo que a função manual de crianças com PC apresenta uma relação moderada e significativa na participação em diferentes áreas, apresentando uma maior correlação da função manual com as atividades instrumentais de vida diária.

Com relação aos resultados obtidos por meio da escala QUEST, observamos melhora em todos os domínios avaliados. Dentre estes, o domínio que avalia os tipos de preensão foi o que teve maior acréscimo na sua pontuação em relação aos outros domínios. Isto foi observado em todos os participantes, exceto no participante 4 que aumentou apenas 3,3 pontos neste domínio e não manteve esse ganho na última avaliação.

Os domínios que avaliaram a dissociação de movimentos e a transferência de peso também apresentaram um acréscimo na pontuação, porém estes foram inferiores quando comparado com o domínio de preensão. Estre resultado corrobora com o estudo de Deluca et al. ${ }^{1}$, onde os autores aplicaram um protocolo de TCI durante 3 semanas em 18 crianças com PC e não observaram diferença significativa no domínio que avalia a dissociação de movimentos da escala Quest. Em contrapartida, Choudhary et al. ${ }^{10}$ aplicaram um protocolo com duração de 4 semanas, e encontraram melhora significativa no domínio que avalia a dissociação de movimentos.

A TCI proporciona uma melhora funcional do membro mais acometido. Além disso, a literatura mostra que esta terapia é capaz promover a ativação de regiões corticais adjacentes à área da lesão ${ }^{23}$. A restrição do membro mais funcional juntamente com a prática intensiva e repetida promove reaprendizado no uso do membro mais afetado e menos funcional, promovendo plasticidade das áreas corticais responsáveis por controlar os movimentos deste membro ${ }^{24}$.

Hoare et al. ${ }^{25}$, realizaram um estudo de revisão com o objetivo de avaliar o efeito da TCI em crianças com PC. A partir dos estudos incluídos nesta revisão, os autores demonstram que a média de duração do protocolo de TCI foi de quatro semanas e a frequência das sessões variou entre dois a sete dias na semana. Além disso os autores apontam 
um risco de viés de moderado a alto entre os estudos. Por fim, concluem que a TCI parece ser melhor para promover ganhos na função uni e bimanual de crianças com PC quando comparada a outras modalidades de "baixa dose", e quando comparada a terapias de "alta dose" os efeitos são similares.

Embora os resultados do presente estudo indicam uma melhora na qualidade do alcance de crianças com PC, ainda se faz necessário a realização de novos estudos para que importante fatores como idade, intensidade, frequência e duração da terapia sejam investigados.

\section{CONCLUSÃO}

Baseados nos resultados obtidos neste estudo, concluímos que o protocolo de TCI realizado parece ter potencial para contribuir com a melhora da função do membro mais acometido, e que estas melhoras podem se manter em até dois meses após o término do protocolo quando comparadas aos valores obtidos antes do treino.

Além disso, observamos que as crianças mais velhas foram mais colaborativas do que as mais novas, desta forma sugerimos que a idade pode ser um fator relevante para os resultados da intervenção e que deve ser levada em consideração para a aplicação do protocolo. No entanto, faz-se necessário mais estudos com um maior número de participantes e ensaios clínicos randomizados para melhor compreensão das variáveis que envolvem esta modalidade de terapia (idade, intensidade, frequência e duração da terapia) para esta população. Novas pesquisas comparando a TCI e seu efeito a longo prazo com outras modalidades de intervenção são necessários para melhor compreensão de sua eficácia.

Agradecimentos: Agradecemos aos participantes e as famílias pela colaboração e por terem aceitado participar do estudo.

Agência de fomento: Agradecemos à Fundação de Amparo à Pesquisa do Estado de São Paulo - FAPESP pelo suporte financeiro (Processo FAPESP n ${ }^{\circ}$ 2015/07438-7).

Contribuição dos autores: Liz Araújo Rohr: Desenvolvimento do projeto, coleta dos dados, análise dos dados e redação do artigo; Camila Araújo Santos Santana: Desenho do projeto, coleta dos dados, análise dos dados e redação do artigo; Erika Shirley Moreira da Silva: Desenvolvimento do projeto, supervisão da coleta dos dados, análise dos dados, aprovação da versão final; Carolina Daniel de Lima-Alvarez: Desenho do projeto e aprovação da versão final; Gabriela Lopes dos Santos-Maia: Análise dos dados e aprovação da versão final; Eloisa Tudella: Desenho do projeto, supervisão do andamento do projeto, escrita e aprovação da versão final.

\section{REFERÊNCIAS}

1. Deluca SC, Echols K, Law CR, Ramey SL. Intensive pediatric constraint-induced therapy for children with the cerebral palsy randomized, controlled, crossover trial. J Child Neurol. 2006;21(11):931-38. doi: 10.1177/08830738060210110401.

2. Beaman J, Kalisperis FR, Miller-Skomorucha K. The infant and child with cerebral palsy. In: Tecklin J. Pediatric physical therapy. 5a ed. Sydney: Lippincott Williams \& Wilkins; 2015. p.187-246.

3. Fonseca LF, Lima CLFA. Paralisia cerebral: neurologia, ortopedia e reabilitação. 2a ed. Rio de Janeiro: Medbook; 2008.

4. Case-Smith J, DeLuca, SC, Stevenson R, Ramey SL. Multicenter randomized controlled trial of pediatric constraintinduced movement therapy: 6-month follow-up. Am J Occup Ther. 2012;1(66):15-23. doi: 10.5014/ajot.2012.002386.

5. Facchin $\mathrm{P}$, Rosa-Rizzotto $\mathrm{M}$, Visonà Dalla Pozza L, Turconi AC, Pagliano E, Signorini S, et. al. Multisite trial comparing the efficacy of constraint-induced movement therapy with that of bimanual intensive training in children with hemiplegic cerebral palsy: postintervention results. Am J Phys Med Rehabil. 2011;90(7):539-53. doi: 10.1097/ PHM.0b013e3182247076.

6. DeLuca SC, Echols K, Ramey SL, Taub E. Pediatric constraintinduced movement therapy for a young child with cerebral palsy: two episodes of care. Phys Ther. 2003;83(11):1003-13. doi: https://doi.org/10.1093/ptj/83.11.1003.

7. Taub E, Uswatte G, Mark VW, Morris DM. The learned nonuse phenomenon: implications for rehabilitation. Eura Medicophys. 2006;42(3):241-55. Available from: https:// www.minervamedica.it/en/journals/europa-medicophysica/ article.php?cod=R33Y2006N03A0241.

8. Taub E, Uswatte G, King DK, Morris D, Crago JE, Chatterjee A. A placebo-controlled trial of constraint-induced movement therapy for upper extremity after stroke. Stroke. 2006;37(4):1045-49. doi: 10.1161/01.STR.0000206463.66461.97. 
9. Charles JR, Wolf SL, Schneider JA, Gordon AM. Efficacy of a child-friendly form of constraint-induced movement therapy in hemiplegic cerebral palsy: a randomized control trial. Dev Med Child Neurol. 2006;48(8):635-42. doi: 10.1017/ S0012162206001356.

10. Choudhary A, Gulati S, Kabra M, Singh UP, Sankhyan N, Pandey RM, et al. Efficacy of modified constraint induced movement therapy in improving upper limb function in children with hemiplegic cerebral palsy: a randomized controlled trial. Brain Dev. 2013;35(9):870-76. doi: 10.1016/j. braindev.2012.11.001.

11. Baleotti LR, Gritti CC, Silva BC. Efeitos de um protocolo modificado da terapia por contensão induzida em criança com paralisia cerebral hemiparética. Rev Ter Ocup Univ São Paulo. 2014;25(3):264-71. doi: 10.11606/issn.2238-6149. v25i3p264-271.

12. Winstein CJ, Miller JP, Blanton S, Taub E, Uswatte G, Morris D, et al. Methods for a multisite randomized trial to investigate the effect of constraint-induced movement therapy in improving upper extremity function among adults recovering from a cerebrovascular stroke. Neurorehabil Neural Repair. 2003;17(3):137-52. doi: 10.1177/0888439003255511.

13. Sakzewski L, Ziviani J, Van Eldik N. Test/retest reliability and inter-rater agreement of the Quality of Upper Extremities Skills Test (QUEST) for older children with acquired brain injuries. Phys Occup Ther Pediatr. 2001;21(2-3):59-67. doi: 10.1080/J006v21n02_05.

14. Rocha NACF, Silva FPS, Tudella E. The impact of object size and regidity on infant reaching. Infant Behav Dev. 2006;29(2):251-61. doi: 10.1016/j.infbeh.2005.12.007.

15. Toledo AM, Soares DA, Tudella E. Proximal and distal adjustments of reaching behavior in preterm infants. J Mot Behav. 2011;43(2):137-45. doi: 10.1080/00222895.2011.552076.

16. Soares DA, van der Kamp J, Savelsbergh GJ, Tudella E. The effect of a short bout of practice on reaching behavior in late preterm infants at the onset of reaching: a randomized controlled trial. Res Dev Disabil. 2013;34(12):4546-4558. doi:10.1016/j.ridd.2013.09.028.
17. Morris DM, Taub E, Mark VW. Constraint-induced movement therapy: characterizing the intervention protocol. Eura Medicophys. 2006; 42: 257-268.

18. Chiu HC, Ada L. Constraint-induced movement therapy improves upper limb activity and participation in hemiplegic cerebral palsy: a systematic review. J Physiother. 2016;62(3):130-37. doi: 10.1016/j.jphys.2016.05.013.

19. Mackey AH, Walt SE, Stott NS. Deficits in upper-limb task performance in children with hemiplegic cerebral palsy as defined by 3-dimensional kinematics. Arch Phys Med Rehabil. 2006;87(2):207-15. doi: 10.1016/j.apmr.2005.10.023.

20. Kreulen M, Smeulders MJC, Veeger HEJ, Hage JJ. Movement patterns of the upper extremity and trunk associated with impaired forearm rotation in patients with hemiplegic cerebral palsy compared to healthy controls. Gait Posture. 2007;25(3):485-92. doi: 10.1016/j.gaitpost.2006.05.015.

21. Machado LR, Heathcock J, Carvalho RP, Pereira ND, Tudella E. Kinematic characteristics of arm and trunk when drinking froam a glass in children with and without cerebral palsy. Clin Biomech (Bristol, Avon). 2019;63:201-6. doi: org/10.1016/j.clinbiomech.2019.03.011.

22. Pashmdarfard M \& Shervin BR. The Impact of Manual Ability Level on Participation of Children with Cerebral Palsy in Life Areas: A Cross-Sectional Study. Iran J Child Neurol. 2019;13(3):83-91. Available from: https://www.ncbi.nlm. nih.gov/pmc/articles/PMC6586446/.

23. Levy CE, Nichols DS, Schmalbrock PM, Keller P, Chakeres DW. Functional MRI evidence of cortical reorganization in upper-limb stroke hemiplegia treated with constraint-induced movement therapy. Am J Phys Med Rehabil. 2001;80(1):4-12. doi: 10.1097/00002060-200101000-00003.

24. Taub E, Uswatte G, Elbert, T. New treatments in neurorehabilitation founded on basic research. Nat Rev Neurosci. 2002;3(3):228-36. doi: 10.1038/nrn754.

25. Hoare BJ, Wallen MA, Thorley MN, Jackman ML, Carey LM, Imms C. Constraint-induced movement therapy in children with unilateral cerebral palsy. Cochrane Database Systematic Rev. 2019(4):CD004149. doi: 10.1002/14651858.CD004149.pub3. 